\title{
Optimal costless extraction rate changes from a non-renewable resource
}

\author{
G. W. EVATT, ${ }^{1}$ P. V. JOHNSON,${ }^{1}$ P. W. DUCK ${ }^{1}$ and S. D. HOWELL ${ }^{2}$ \\ ${ }^{1}$ School of Mathematics, University of Manchester, Manchester, M13 9PL, UK \\ email : geoffrey.evatt@manchester.ac.uk \\ ${ }^{2}$ Manchester Business School, University of Manchester, Booth Street West M15 6PB, UK
}

(Received 21 March 2013; revised 4 July 2014; accepted 8 July 2014; first published online 4 August 2014)

This paper considers the role of costless decisions relating to the extraction of a non-renewable resource in the presence of uncertainty. We begin by deriving a size scale of the extractable resource, above which the solution to the valuation and optimal control strategy can be described by analytic solutions; we produce solutions for a general form of operating cost function. Below this critical resource size level the valuation and optimal control strategy must be solved by numerical means; we present a robust numerical algorithm that can solve such a class of problem. We also allow for the embedding of an irreversible investment decision (abandonment) into the optimisation. Finally, we conduct experimentation for each of these two approaches (analytical and numerical), and show how they are consistent with one another when used appropriately. The extensions of this paper's techniques to renewable resources are explored.

Key words: Real Options; Mining; Optimal Control; Decision under Uncertainty; SemiLagrangian

\section{Introduction}

The purpose of this paper is to help the operator of a non-renewable resource optimise the rate of extraction when the future commodity price is uncertain. We primarily consider the class of extraction rate control that can be altered without any sunk capital investment, such as those associated with variable capacity rates (Slade, 2001). We shall refer to this type of control as a costless decision, which is in contrast to a irreversible decision (such as a processing plant expansion) which cannot be undone without loss of capital investment (Pindyck, 1988; Hanemann, 1989). Irreversible decision making forms the backbone of Real Options Analysis and has been frequently applied to non-renewable extraction project analysis (e.g. Brennan \& Schwartz, 1985; Evatt et al., 2011). Yet the analysis of costless control strategies has been relatively unexplored (e.g. (Cherian et al., 2000; Kamrad \& Earnst, 2001)) and it was recognised by Cherian et al. (2000), Slade (2001) and Benchekroun et al. (2009) ${ }^{1}$ that a larger investigation into costless control strategies was required.

1 Here they refer to endogenously determined extraction rate changes as 'closed-loop' strategies. 
Classic studies of the extraction of non-renewable natural resources have also frequently assumed that the size of the resource is 'large enough' for its valuation to be the same as the value associated with an infinite resource (e.g. Brennan \& Schwartz, 1985; Dixit \& Pindyck, 1994). Indeed it is often extremely convenient to assume infinite resources because it can allow exact analytic solutions to the valuation problem to exist (because the mathematical structure can be reduced to an ordinary differential equation). However, to assume infinite resources may unrealistically simplify the problem: how can we recognise when the remaining resource is not 'large enough' for the classic solution to give an acceptable approximation (McCarthy \& Monkhouse, 2002)? To rectify this outstanding problem we use a simple scaling approach to present an estimate of the critical size level of the resource beyond which one can justify the assumption of an infinite resource and therefore use analytical solution techniques. When the size of the resource is below this level one should solve the full underlying model and thus resort to computational techniques so as to extract optimal solutions. To help practitioners achieve this, we present an effective and accurate algorithm based upon an iterative semi-Lagrangian numerical procedure for determining solutions to the full model. As such, this critical resource size estimate may be used by interested parties to provide information (and confidence) as to the strength and appropriateness of these different solution approaches.

We have chosen to primarily consider commodity price uncertainty within our modelling because it is (generally) an exogenous quantity and is frequently found to be the dominant uncertainty within an extraction project (Dominy et al., 2002; Moel \& Tufano, 2002; Deloitte, 2011; Evatt et al., 2012). It also provides a common foundation to build costless decision-making analysis upon because price uncertainty has been widely considered within irreversible decision-making studies. That said, clearly the ultimate goal of this area of work is to appropriately amalgamate all of the key uncertainties an extraction project faces (Atkinson \& Isangulov, 2010), such as the consideration of uncertain operating costs (Dehghani \& Ataee-pour, 2012) or the inclusion of natural growth (or refilling) of the resource (Provencher, 1995; Sarkar, 2000; Singh et al., 2006). We discuss these potential extensions of our work within Section 6.

The remaining structure of this paper is as a follows. In Section 2, we develop the mathematical model which is used to determine both the valuation and the optimal control strategy. We then explore this further in Section 3, by deriving the criteria for when one can consider the resource 'large enough' to be approximated as infinite. We then use this criterion to extract analytical solutions to the valuation and control strategies in Section 4. In Section 5, we present and develop the numerical scheme which can solve the full mathematical model for smaller resources, before detailing results from the scheme. We conclude our work in Section 7.

\section{Mathematical formulation}

To determine the valuation, $V$, of a nonrenewable resource subject to costless controls within a certain solution domain $H$, we first prescribe the state-space variables. We are considering just one uncertainty, the price per unit of the underlying $S_{t}$, along with the current resource size $Q_{t}$, and time $t$. As such, $H \in \mathbf{R}^{3}$. 
We may assume that the underlying commodity price $S_{t}$ follows an Itô diffusion of the form,

$$
d S_{t}=\hat{\mu}\left(S_{t}, t\right) d t+\hat{\sigma}\left(S_{t}, t\right) d X
$$

where $\hat{\mu}$ is the drift of the price, $\hat{\sigma}$ is its percentage volatility and $X$ is a standard Wiener process. Within this paper, we make the non-restrictive assumption that the price process follows a risk-neutral ${ }^{2}$ Geometric Brownian Motion (GBM), and thus $\hat{\mu}=(r-\delta) S$ and $\hat{\sigma}=\sigma S$, where $\sigma$ is the percentage volatility, $r$ is the discount rate and $\delta$ is the convenience yield (representing market effects such as those associated with the delivery cost of a commodity Brennan, 1958). Interestingly, in Section 3, we find that the approximate optimal control strategy for extraction, is independent of the particular form of the underlying price process (although the associated valuation is clearly altered).

The rate of extraction of the resource, $q=q\left(S_{t}, Q_{t}, t\right)$, is introduced via the (trivial) stochastic differential equation

$$
d Q_{t}=-q d t
$$

and will be subject to physical and practical constraints on maximum $\bar{q}$ and minimum $\underline{q}$ levels, requiring $q \in[q, \bar{q}]$ for all time. It is this extraction rate $q$, that is the control variable within the operating system. The extraction rate will have an associated cost function whose form, we assume, is known to the resource owners and we denote it by $\epsilon=\epsilon\left(q, Q_{t}, t\right)$. As such, within a given increment of time, the rate of (pre-tax) cash flows that can be generated from the extraction project can be written as

$$
q S_{t}-\epsilon
$$

By defining the extraction project owner to be interested in maximising the total expected future discounted cash flows, we can write the valuation of the extraction operation as

$$
V\left(S_{t}, t, Q_{t}\right)=\sup _{q}\left(E_{x} \int_{t}^{v^{*}}\left(q S_{v}-\epsilon\right) e^{-r v} d v\right)
$$

where $v^{*}$ is the time at which extraction ceases and $E_{x}$ denotes the expected value when $\left(S_{t}, t, Q_{t}\right)=x \in \mathbf{R}^{3}$.

With the integral (2.4) and processes (2.1) and (2.2), we may utilise the well-known Hamilton-Jacobi-Bellman (HJB) equation (Øksendal, 2003) to derive the associated partial differential equation (PDE) which holds within the domain $H$ :

$$
\frac{\partial V}{\partial t}+(r-\delta) S \frac{\partial V}{\partial S}+\frac{1}{2} \sigma^{2} S^{2} \frac{\partial^{2} V}{\partial S^{2}}-r V+\sup _{q}\left(-q \frac{\partial V}{\partial Q}+S q-\epsilon\right)=0
$$

2 We have chosen to use a risk-neutral measure so as to be in keeping with much of the Real Options literature. Whilst this assumption will not always be the appropriate probability measure to use (e.g. in the absence of continual hedging), we may nonetheless use it without loss of generality. To switch this paper's mathematics back to real-world measure of GBM, one simply would have to make the substitution $\delta=r-\mu$, where $\mu$ is the real-world percentage drift. More discussion upon this point in given in Wilmott (2009). 
where this PDE representation no longer uses time subscripts, and we have assumed $q S_{v}-\epsilon$ is sufficiently bounded for a viscosity solution to exist.

We next need to prescribe boundary conditions to (2.5). If an extraction company has a lease to extract, then at the contract termination date, $T$, its value will be zero, i.e.

$$
V=0 \quad \text { at } \quad t=T \text {. }
$$

The boundary condition relevant to the level of resource size is that when the extractable amount of resource is exhausted, no more economic value exists, and so

$$
V=0 \quad \text { on } \quad Q=0
$$

Since the extraction rate has a physical upper bound, the extraction rate and cost will behave as if independent of $S$ when $S$ is large. This permits a far field valuation of the form,

$$
V \rightarrow A(Q, t) S+B(Q, t) \quad \text { as } \quad S \rightarrow \infty .
$$

When the underlying commodity becomes worthless we are left to solve a reduced form of equation (2.5), with $S=0$.

\section{How large is 'large enough'?}

We now examine equation (2.5) to see which meaningful analytical 'order of magnitude' information can be extracted. Clearly, the presence of the $\frac{\partial V}{\partial Q}$ term within the supremum complicates matters, for otherwise solutions to the optimal strategy are uncoupled from it. Indeed, it is in effect the setting of $\frac{\partial V}{\partial Q}=0$ which allowed Brennan \& Schwartz (1985) and many others (e.g. Dixit \& Pindyck, 1994; Cortazar et al., 1998) to extract closed-form solutions to their Real Options valuation models related to nonrenewable resources. This simplification is made by assuming that the resource is 'large enough' (often crudely referred to as 'infinite'), that the sensitivity of the valuation to resource size is negligible. The assumption can be used to great effect in helping render solutions tractable. However, within the context of resource management the size criterion that makes it permissible to neglect the derivatives in $t$ and $Q$ has not yet been explored. As such, we need to derive an estimate for when a resource can be considered 'large enough' so that we can employ solution techniques appropriately.

We first note that we would expect an extraction company to commence operation only once there is a positive value in doing so, i.e. when the commodity price is sufficiently high. In the limit, this is the valuation given by (2.8) and it is also the value given in the absence of price uncertainty $(\sigma=0$ in (2.8)). As such, we shall use it as our typical order of magnitude size-scale for the valuation, where it is given by

$$
V=\frac{\hat{q} S}{\delta}\left(1-e^{-\delta(T-t)}\right)-\frac{\epsilon}{r}\left(1-e^{-r(T-t)}\right) .
$$

We see from the above relation that the time-scale beyond which it is largely insensitive to variations in time, is for contract periods of size $O(1 / \delta)$. This time-scale can be used as that for which time-derivatives no longer play a dominant role within (2.5), thus providing 
the criteria for a long enough contract duration:

$$
\tilde{T}=\frac{1}{\delta}
$$

This particular time-scale can then be matched via (2.2) to the resource size-scale $\tilde{Q}$ which marks the typical size beyond which the valuation is also insensitive to derivatives in $Q$ :

$$
\tilde{Q}=\frac{\hat{q}}{\delta}
$$

where we have chosen the extraction rate size scale as its maximum rate $\hat{q}$. As a consequence, we shall refer to $\tilde{Q}$ as the large enough resource size.

It is to be noted that our time-scale is different from those associated with, say, the Black-Scholes equation for financial options where the time scale has terms in $\sigma^{-2}$ (Ahn et al., 2006). This distinction arises from the fact that the size order of cash flows plays a dominant role in the valuation of a extraction project; as previously mentioned, one expects an operator to invest in extraction projects only when the cash-flows are positive. This should be seen in contrast to financial options, where options are frequently created 'out of the money' (primarily for hedging reasons) and generally lack cash flows (since value arises only at option expiry).

\section{Large enough resource analysis}

\subsection{Optimal control for 'large enough' resources}

Before we make use of the 'large enough' approximation, let us consider where the optimization of the control strategy arises. The control strategy that maximises the valuation $V$ is clearly determined by equation (2.5). In particular, the optimal control strategy stems from the term

$$
\sup _{q}\left(-q \frac{\partial V}{\partial Q}+S q-\epsilon\right),
$$

and we denote the optimal feasible strategy by $q^{*}$. To find $q^{*}$, we shall assume the above equation is sufficiently smooth in $q$ and thus differentiate it with respect to the control strategy (and since the strategy is optimal, we know $\frac{\partial V}{\partial q}=0$ along $q=q^{*}$ ). This provides the control equation

$$
-\frac{\partial V}{\partial Q}+S-\frac{\partial \epsilon}{\partial q}=0
$$

which must be satisfied so as to ensure optimality within the feasible region $q^{*} \in[q, \bar{q}]$. If the optimal solution is not within the feasible region, one would have to choose $\bar{q}^{*}$ to either be $\underline{q}$ or $\bar{q}$, depending upon which one satisfies (4.1).

As previously mentioned, finding a closed-form optimal solution using (4.2) is difficult due to the presence of the undetermined term $\frac{\partial V}{\partial Q}$. However, thanks to the results of the previous section we now have a firm test of when it is permissible to neglect this term. On 
setting $\frac{\partial V}{\partial Q}=0$ in equation (4.2), we may solve (4.2) for an approximate $q^{*}$ as the root of

$$
\frac{\partial \epsilon}{\partial q}\left(q^{*}, Q, t\right) \approx S
$$

This simple approximation gives powerful insights into the optimal extraction regime. For example, if the cost function behaves as a power law of the form $\epsilon=a q^{n}$, where $a$ and $n>1$ are constants, then our large-resource optimal extraction rate would behave as

$$
q^{*} \approx \max \left\{\underline{q}, \min \left[\left(\frac{S}{n a}\right)^{\frac{1}{n-1}}, \bar{q}\right]\right\} .
$$

If a solution for (4.3) does not exist (for example if $\epsilon$ is constant or linear with respect to $q$ ), then $q^{*}$ must equal one of its bounds, $\bar{q}$ or $\underline{q}$. As such, the approximation of (4.3) gives valuable insight into when the structure of the optimal regime will be bang-bang and when it will not. It is notable that (4.3) contains no terms from the price-time process (i.e. $r, \delta$ and $\sigma$ are absent). This is because the control variable $q$ has no dynamics, and if $t$ and the resource $Q$ are both infinite, $q^{*}$ needs to exercise no foresight when consuming $Q$. Hence, the instantaneous choice of $q^{*}$ need only maximise cash flow within each time increment $d t$. Of course the expected effects of the future dynamics of $S$ itself, along with the time value of money, are present in the full PDE for $V(2.5)$, which must be solved after maximising $S q-\epsilon$ at every $S$ state point.

\subsection{Optimal valuation for large enough resources}

We now seek to determine the valuation of a 'large enough' resource, where we can use the approximate optimal control strategy given by (4.4) and assume there is no time limit to the extraction lease. For notational convenience, we set $a=\bar{q} / \bar{\epsilon}$ to rewrite the cost function as

$$
\epsilon=\bar{\epsilon}\left(\frac{q}{\bar{q}}\right)^{n},
$$

where $\bar{\epsilon}$ is a prescribed constant maximum running cost. Further, we shall make the convenient assumption that $q=0$, thus allowing the company to no longer operate when the commodity has become worthless. By substituting this cost function into the optimal control strategy given by (4.4), and inserting this into the equation (2.5) (where we impose $\frac{\partial V}{\partial Q}=\frac{\partial V}{\partial t}=0$ ), we find

$$
\frac{1}{2} \sigma^{2} S^{2} \frac{\partial^{2} V}{\partial S^{2}}+(r-\delta) S \frac{\partial V}{\partial S}-r V+q^{*} S-\epsilon\left(q^{*}\right)=0 .
$$

To aid in solving this equation, we split our solution domain into two complementary regions: an upper region (denoted by the ' + ' superscript) where the extraction rate is at its maximum value, and a lower region (denoted by the '-' superscript) where the extraction rate is continuously variable. The meeting point of these two regions, $\bar{S}$, must 
be determined via matching terms in (4.4), so that

$$
\bar{S}=\frac{n \bar{q}^{n}}{\bar{\epsilon}} .
$$

At this point of intersection, the two regions' valuations must meet smoothly, thus supplying us with the final two boundary conditions to close the model.

In the upper region, we know that $q^{*}$ is given by the constant value $\bar{q}$, and so one can extract from (4.6) the general solution

$$
V^{+}=A^{+} S^{\alpha_{1}}+B^{+} S^{\alpha_{2}}+\frac{\bar{q} S}{\delta}-\frac{\bar{\epsilon}}{r}
$$

where $A^{+}$and $B^{+}$are constants and

$$
\alpha_{1,2}=\frac{1}{2}-\frac{r-\delta}{\sigma^{2}} \pm\left[\left(\frac{1}{2}-\frac{r-\delta}{\sigma^{2}}\right)^{2}+\frac{2 r}{\sigma^{2}}\right]^{1 / 2}
$$

where we always have freedom to specify $\alpha_{1}<0$ and $\alpha_{2}>0$.

In the lower (continuously variable) region, the general solution is given by the form

$$
V^{-}=A^{-} S^{\alpha_{1}}+B^{-} S^{\alpha_{2}}+h(S),
$$

where $A^{-}$and $B^{-}$are both constants (to be determined) and

$$
\begin{aligned}
h(S) & =\phi S^{\gamma}, \\
\phi & =\left(\frac{\beta-a \beta^{n}}{r-(r-\delta) \gamma+\gamma(1-\gamma) \sigma^{2} / 2}\right), \\
\beta & =\left(\frac{\bar{q}}{n \bar{\epsilon}}\right)^{\frac{1}{n-1}}, \\
\gamma & =\frac{n}{n-1} .
\end{aligned}
$$

By noting that as $S$ becomes smaller, the valuation cannot increase, we must have $A^{-}=0$. Equally, as the price grows large the valuation must grow linearly in price, implying $B^{+}=0$. Next we may utilise the fact that the two regions must smoothly meet at the known point $\bar{S}$, which is to say:

$$
\begin{aligned}
V^{+}(\bar{S}) & =V^{-}(\bar{S}), \\
\frac{\partial V^{+}}{\partial S}(\bar{S}) & =\frac{\partial V^{-}}{\partial S}(\bar{S}) .
\end{aligned}
$$

By using the above conditions, we can solve for the unknown parameters in (4.10) and (4.11) to find

$$
\begin{aligned}
& V^{+}=A^{+} S^{\alpha_{1}}+\frac{\bar{q} S}{\delta}-\frac{\bar{\epsilon}}{r}, \\
& V^{-}=B^{-} S^{\alpha_{2}}+h(S),
\end{aligned}
$$


where

$$
\begin{aligned}
& A^{+}=\frac{\bar{S}^{-\alpha_{1}}}{\alpha_{1}-\alpha_{2}}\left[\bar{S} h^{\prime}(\bar{S})-\alpha_{2} h(\bar{S})-\frac{\alpha_{2} \bar{\epsilon}}{r}-\frac{\bar{q} \bar{S}\left(1-\alpha_{2}\right)}{\delta}\right], \\
& B^{-}=\frac{\bar{S}^{-\alpha_{2}}}{\alpha_{1}-\alpha_{2}}\left[\bar{S} h^{\prime}(\bar{S})-\alpha_{1} h(\bar{S})-\frac{\alpha_{1} \bar{\epsilon}}{r}-\frac{\bar{q} \bar{S}\left(1-\alpha_{1}\right)}{\delta}\right] .
\end{aligned}
$$

This closed-form solution gives us the valuation associated with a (large enough) extraction project obeying the control strategy of (4.4), subject to a cost function of the form (4.5) with $n>1$.

There are two notable instances when the closed form solution of (4.13) breaks down, and that is when the costs are either constant $(n=0)$ or linear in the extraction rate $(n=1)$. In the latter instance, one merely sets $h=0$ in (4.13) and (4.14) to find the associated closed form solution. In the constant running cost instance $(n=0)$, the firm is continually exposed to a fixed cost rate and may never alter its value. As such, one may consider the cost as a perpetually paid cash flow, and thus one would set $h=-\epsilon / r$ in (4.13) and (4.14).

\subsection{Hybrid decisions}

With the basis of our analysis of costlessly variable decision making developed, we may now embed an irreversible decision within the extraction project. We assume that the operator acts rationally and abandons the operation altogether if the value of the operation becomes sufficiently low (where the operator is indifferent between abandonment and continuing operation). If we suppose the associated close-down cost is some known value $K$, then abandonment occurs when $V=-K$. However, in order for our prior modelling to permit a negative valuation we clearly need to add in an extra parameter: fixed running costs $\epsilon_{f}$. Since these fixed costs cannot be reduced by varying the extraction rate, it is clear that for low enough commodity prices, the valuation shall become negative, and thus the possibility exists that it is cost-effective for the operation to abandon operation altogether (Brennan \& Schwartz, 1985).

In a similar manner to solving the previous section, we must smoothly match the valuation of the separate regions (abandoned region, operational region) when the price is at its optimal abandonment level $S_{a}$. In such irreversible investment problems, the optimal abandonment price is a free-boundary, which must be found as part of the solution of the system. As such the number of free parameters in this problem grows, and the required algebraic calculations grow much further still. To make matters (significantly) more manageable, we shall presently assume there is no upper limit to $q$ and thus we need only consider solving the problem in two regions ${ }^{3}$.

3 That said, to find a solution to the problem of a finite $\bar{q}$ one could simply write down the smooth pasting equations for the model in the three regions and use a basic numerical iteration to find the (five) corresponding parameter values. 
By following precisely the same solution methodology as the previous section (smooth matching of the valuations at the abandonment price), we may determine our solution as,

$$
V=A S^{\alpha_{1}}+h(S)-\frac{\epsilon_{f}}{r}
$$

where

$$
\begin{aligned}
A & =-\frac{\gamma \phi S_{a}^{\gamma-\alpha_{1}}}{\alpha_{1}} \\
S_{a} & =\left(\frac{\epsilon_{r}}{r}-K\right)^{1 / \gamma} \phi^{-1 / \gamma}\left[1-\frac{\gamma}{\alpha_{1}}\right]^{-1 / \gamma} .
\end{aligned}
$$

As this solution highlights, in order for the abandonment price to exist, the condition that $\epsilon_{r} / r>K$ must hold.

\subsection{Large resource results}

With our closed-form solution derived for a 'large enough' resource (where the extraction cost assumption is given by (4.5)) we explore the overall behaviour of the model. We apply these models to a hypothetical oil well whose size is measured in terms is barrels, measured in units b. For transparency and without loss of generality, we prescribe parameter values given by Schwartz (1997), which were collected over a five year period from oil futures contracts. We make our own reasonable assumptions regarding the maximum extraction rate and associated costs. Unless we specify otherwise, these parameter values are given by

$$
\begin{aligned}
\delta=0.17 \mathrm{yr}^{-1}, & r=0.05 \mathrm{yr}^{-1}, \quad \bar{q}=10^{6} \mathrm{~b} \mathrm{yr}^{-1} \\
\sigma=0.34 \mathrm{yr}^{-\frac{1}{2}}, & \bar{\epsilon}=2 \times 10^{7} \$ \mathrm{yr}^{-1}, \quad \mathrm{~K}=10^{7} \$ .
\end{aligned}
$$

It is important to note that our results do not include considerable upfront investment costs, nor do we explicitly include taxes (although one might consider the latter as part of the cost function), and thus we are finding the present value of an existing pre-tax flexible operation, not the total Net Present Value of an operation yet to be invested in.

We first calculate the optimal extraction regime (4.4) and associated cost function (4.5) for three different values of $n$. The results for the cost function and optimal extraction rate are shown in Figure 1 bottom and top, respectively. The continuous line corresponds to $n=1$, the dotted line corresponds to $n=2$ and the dashed line corresponds to $n=3$. With these optimal control strategies and costs we can calculate the corresponding valuation via equation (4.13), whose results are shown in Figure 2 (with the same markings for each set of results). The results show a monotonic increase in valuation with an increase in $n$, a consequence of more widely variable operating costs, as highlighted in Figure 1 (bottom).

We next explore the effects of varying the maximum extraction cost $\bar{\epsilon}$, for a fixed value of $n=2$. The results for the costs and optimal extraction rate are shown in Figure 3 (bottom and top, respectively), where the dashed line has $\bar{\epsilon}=10^{7} \$ \mathrm{yr}^{-1}$, the continuous line has $\bar{\epsilon}=2 \times 10^{7} \$ \mathrm{yr}^{-1}$ and the dotted line is the most costly, at $\bar{\epsilon}=3 \times 10^{7} \$ \mathrm{yr}^{-1}$. These maximum extraction costs correspond to $\bar{S}=20,40,60 \$ \mathrm{~b}^{-1}$ respectively. Using 

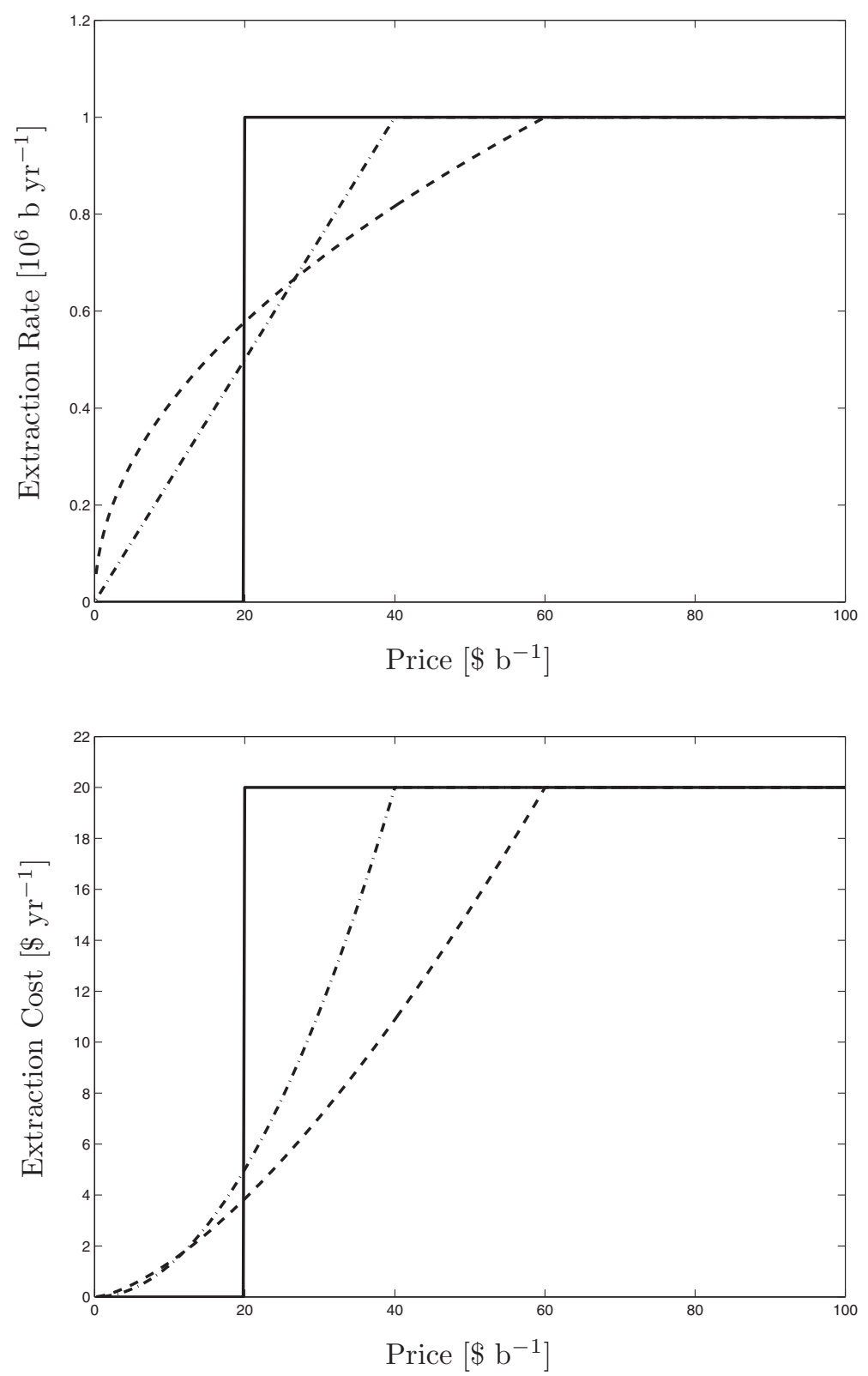

Figure 1. The optimal extraction rate (top) and cost function (bottom) for three different values of $n$ in the cost function (4.5): the continuous line has $n=1$, the dotted line has $n=2$ and the dashed line has $n=3$.

the costs and optimal extraction regimes as shown in Figure 3, we now solve (4.13) for their corresponding valuations. The results are shown in Figure 4 (with the same line markings as the costs in Figure 3). As one would expect, the valuations tend to zero for small costs, and rapidly tend to a linear function for larger values of price. 


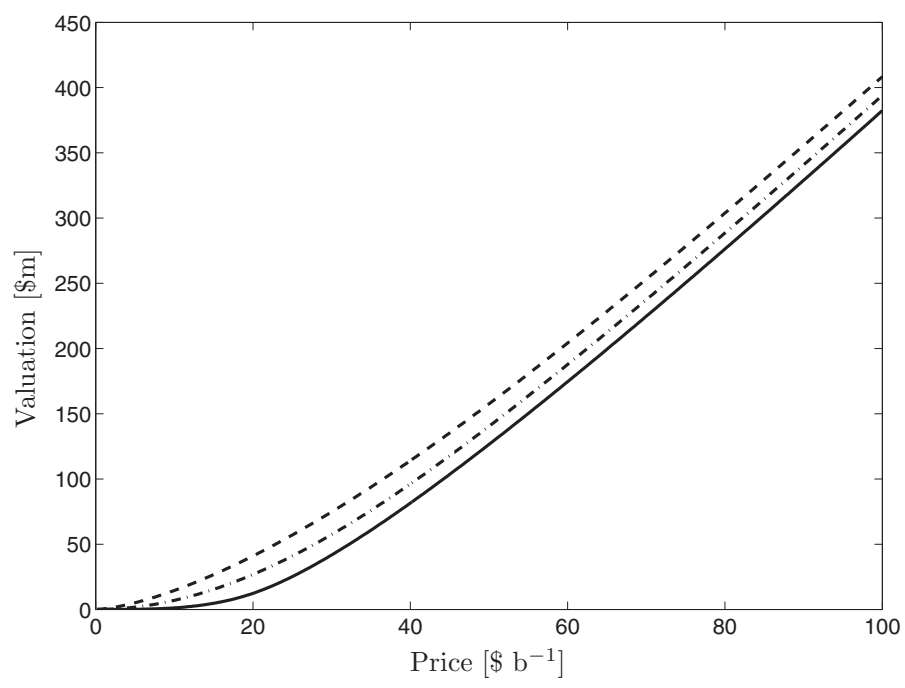

FIGURE 2. Three maximal valuations for a 'large enough' resource, corresponding to the three different optimal extraction rates and costs as shown in Figure 1, in which the continuous line has $n=1$, the dotted line has $n=2$ and the dashed line has $n=3$. Calculated from equation (4.13).

To highlight the sensitivity of the value of the flexible operation to the price volatility, Figure 5 shows the value for three different values of $\sigma$ (all with quadratic cost functions). From bottom to top: $\sigma=24 \%, 34 \%, 44 \% \mathrm{yr}^{-\frac{1}{2}}$. As the results show for this particular set of parameter values, the absolute differences between the valuations remain roughly constant for all prices above approximately $40 \$ \mathrm{~b}^{-1}$. This observation ties in with the fact that the operation is always extracting at its constant maximum capacity for prices above this level.

The final results of this section are for when we include the option to abandon the project, which occurs when the prices becomes sufficiently low. We plot the solutions of equation (4.15) in Figure 6, for three different values of fixed running cost, from left to right: $\epsilon_{r}=\$ 10^{7} \mathrm{yr}^{-1}, \epsilon_{r}=\$ 2 \times 10^{7} \mathrm{yr}^{-1}$ and $\epsilon_{r}=\$ 3 \times 10^{7} \mathrm{yr}^{-1}$. As would be expected, the abandonment position (where the solution gradient becomes flat) increases for higher running costs, when the project is less cost effective. Since our solutions for the abandonment case do not have a restriction upon the upper extraction rate $\bar{q}$, the valuations for larger prices are significantly higher than for previous results where there was such a restriction.

\section{Full model investigation}

In models which omit dependence on $Q$ and $t$ by assuming a 'large enough' reserve, we have shown that closed-form solutions may be obtained, provided one specifies the functional form of the extraction costs $\epsilon(q)$. However, it is clear that such an approximation will often not be appropriate (where our derived scaling laws of (3.3) and (3.2) may be used to determine this appropriateness), and in these instances one will instead have to resort to numerical means to extract information. To this end, whilst a Monte-Carlo approach 

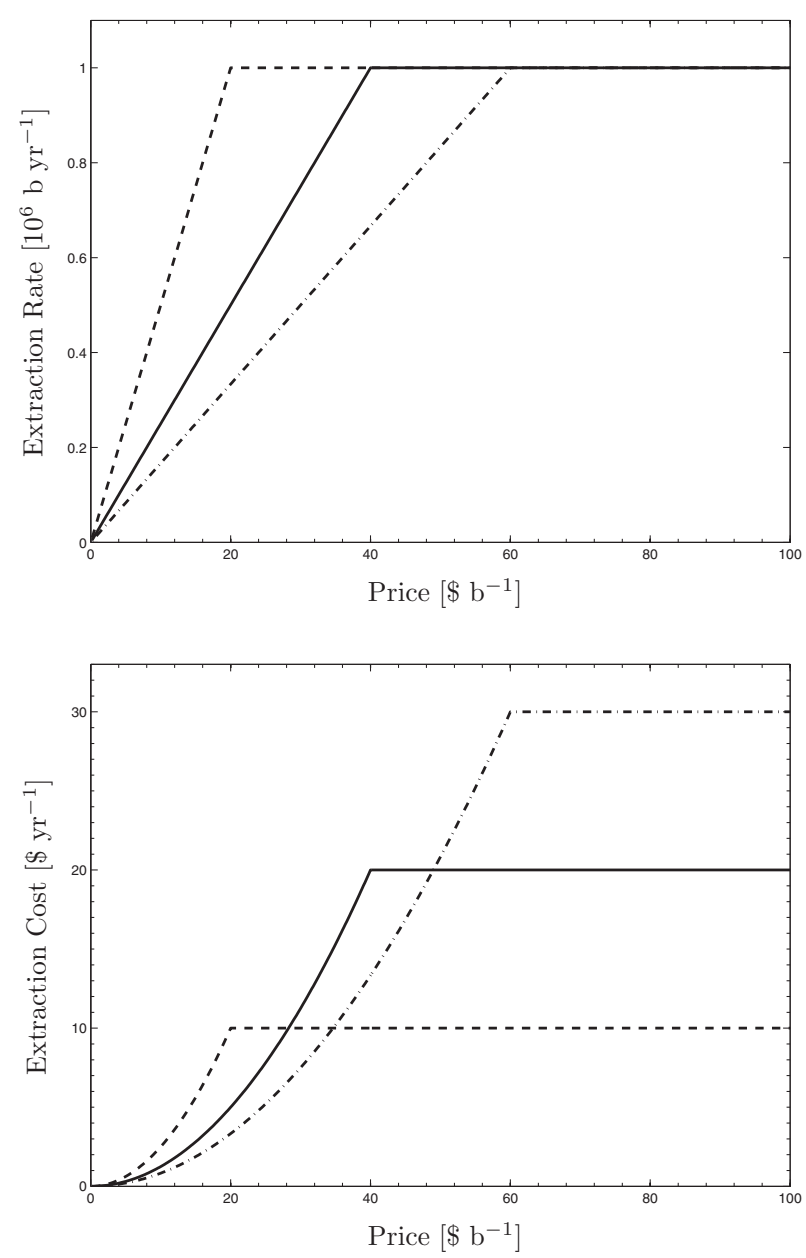

Figure 3. The optimal extraction rate (top) and associated cost function (bottom) using three different maximum extraction costs for a quadratic cost function $(n=2)$. The dashed line has $\bar{\epsilon}=10^{7} \$ \mathrm{yr}^{-1}$, the continuous line has $\bar{\epsilon}=2 \times 10^{7} \$ \mathrm{yr}^{-1}$ and the dotted line has $\bar{\epsilon}=3 \times$ $10^{7} \$ \mathrm{yr}^{-1}$.

might be tempting to use (primarily due to its simplicity), it does not lend itself so readily to our optimisation problem as a PDE numerical approach naturally does: PDEs can be constructed so as to solve exactly for maxima; there is consistently high accuracy in the PDE results; significant increases in computational speed are given for this class of problem; numerical PDE solution methodologies are transparent for objective criticism and replicable by third parties. As such, we shall use a PDE approach to solve our optimisation problem for smaller resource sizes.

\subsection{Numerical method}

Solving the optimisation problem of equation (2.5), for a resource smaller than the 'large enough' size limit, is relatively complex. This is because the solution for $q^{*}$ is dependent 


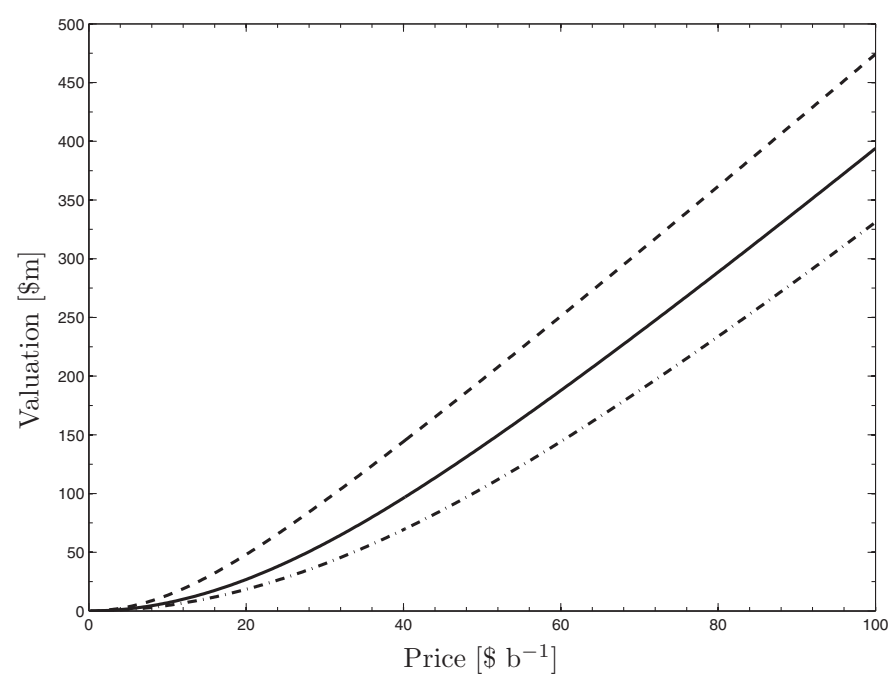

FIGURE 4. Three maximal valuations corresponding to the three different quadratic cost functions, as shown in Figure 3, in which the dashed line has $\bar{\epsilon}=10^{7} \$ \mathrm{yr}^{-1}$, the continuous line has $\bar{\epsilon}=2 \times 10^{7} \$ \mathrm{yr}^{-1}$ and the dotted line has $\bar{\epsilon}=3 \times 10^{7} \$ \mathrm{yr}^{-1}$. Calculated from equation (4.13).

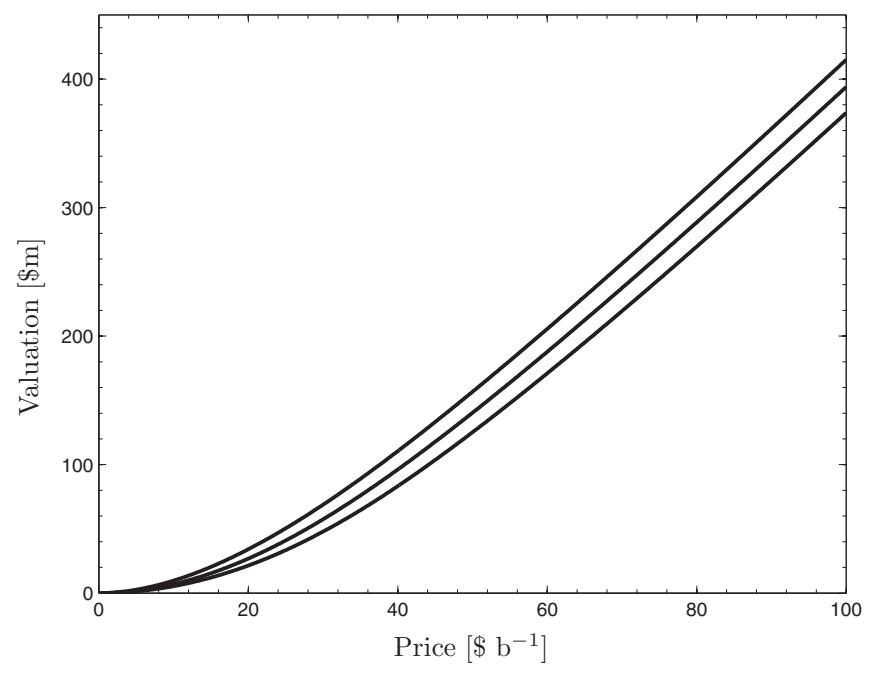

FIGURE 5. The maximal valuation for a quadratic cost function, in which the price volatility is varied: bottom line $\sigma=24 \% \mathrm{yr}^{-\frac{1}{2}}$, middle line $\sigma=34 \% \mathrm{yr}^{-\frac{1}{2}}$, and top line $\sigma=44 \% \mathrm{yr}^{-\frac{1}{2}}$. Calculated from equation (4.13).

upon $V^{*}$, which itself can only be calculated once $q^{*}$ has been found. Thus, it appears that an iterative process is required to solve this inherently nonlinear problem, making it a challenging form of dynamic programming. However, there is a technique to treat this nonlinearity, and we show how using a semi-Lagrangian method (Staniforth \& Cote, 1991) can reduce the problem to an optimisation followed by a linear solve. Other papers addressing related problems emit solutions of the so-called bang-bang type, meaning that there are only a finite set of optimal choices, essentially arising from the use of a linear 


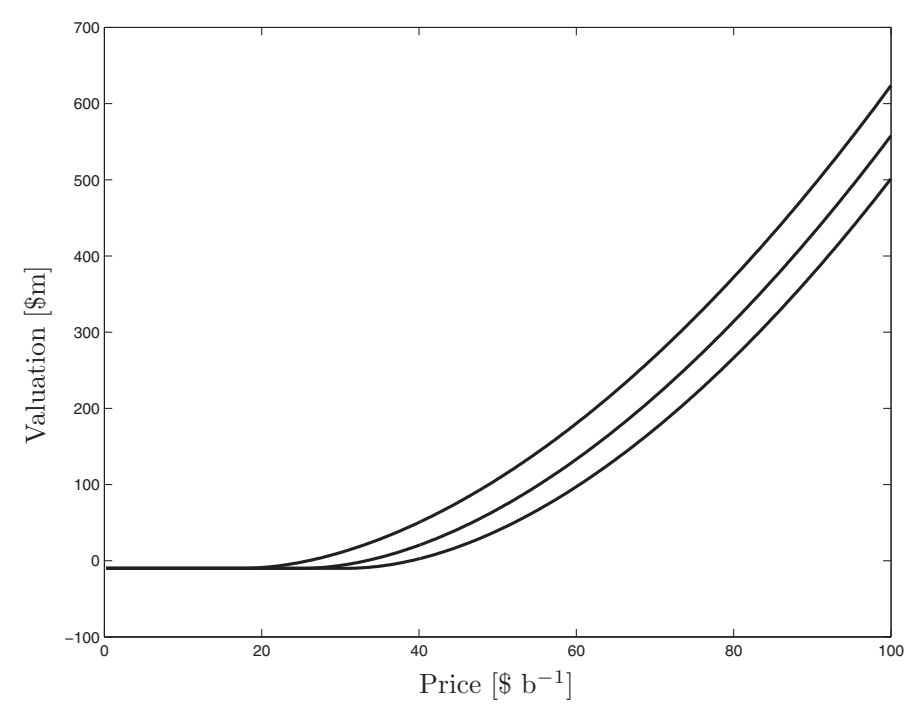

FIGURE 6. The maximal valuation for a quadratic cost function complete with the option to abandon, in which the fixed running cost is varied. From left to right: $\epsilon_{r}=\$ 10^{7}, \epsilon_{r}=\$ 2 \times 10^{7}$ and $\epsilon_{r}=\$ 3 \times 10^{7}$. Calculated from equation (4.15).

cost function (Chen \& Forsyth, 2007; Evatt et al., 2011). In similar notation, we present the numerical approach which takes account of a much wider range of possible cost functions.

The boundary conditions in this problem admit solutions with a discontinuity in $\frac{\partial V}{\partial Q}$, which can cause errors to propagate and amplify throughout the system. To remove such issues we choose to place the grid nodes of the numerical scheme where they coincide precisely with the characteristics. Using the standard notation of $\tau=T-t$ to represent the time left to maturity, consider the path (known as the semi-Lagrangian trajectory) through the domain $Q \times \tau$ which satisfies the equation

$$
\frac{d Q}{d \tau}=\bar{q}
$$

This characteristic direction can be expressed as $Q=\bar{q} \tau$, where we have made use of (2.7). Since the extraction rate is bounded by $\bar{q}$, we can say that in the region above this line $(Q>\bar{q} \tau)$ the resource cannot be emptied before the contract expires, so that trajectories in this region are no longer functions of $Q$, only of $\tau$. We therefore choose the grid spacing $\Delta Q=\bar{q} \Delta \tau$, so that there is always a node on the line $Q=\bar{q} \tau$ which separates the two regions.

Let us introduce the operator $\mathscr{L}$, which is given by

$$
\mathscr{L}\{V\} \equiv \frac{1}{2} \sigma^{2} S^{2} \frac{\partial^{2} V}{\partial S^{2}}+(r-\delta) S \frac{\partial V}{\partial S}-r V .
$$

Once the optimal choice of $q$ has been prescribed, it allows us to more succinctly write 
equation (2.5) as

$$
\frac{\partial V}{\partial \tau}+q \frac{\partial V}{\partial Q}=\mathscr{L}\{V\}+q S-\epsilon .
$$

By considering a path through $Q \times \tau$ which satisfies the equation

$$
\frac{d Q}{d \tau}=q
$$

we may define the left-hand-side of (5.3) as

$$
\frac{D V}{D \tau}=\frac{\partial V}{\partial \tau}+\frac{d Q}{d \tau} \frac{\partial V}{\partial Q}
$$

and hence

$$
\frac{D V}{D \tau}=\mathscr{L}\{V\}+q S-\epsilon .
$$

This form of equation allows us to make use of the semi-Lagrangian numerical method.

We choose to discretise the system with an equally spaced grid in $S, Q$ and $\tau$ so that $S_{i}=i \Delta S, Q_{j}=j \Delta Q$ and $\tau_{k}=k \Delta \tau$. While the right-hand-side of equation (5.6) can be expressed with standard finite differences at nodes in the grid, the left-hand-side may in general take values not located on a standard grid node, so that values at the latter will require interpolation. If we assume a Crank Nicolson scheme taking finite difference approximations at the half timestep, at the node $(i, j, k)$ we have $V_{i, j}^{k}=V\left(S_{i}, Q_{j}, \tau_{k}\right)$ and $q_{i, j}^{k}=q\left(S_{i}, Q_{j}, \tau_{k}\right)$, then in order to evaluate the total derivative on the left-hand-side we need to find the path in $Q$ that arrives at the point $Q_{j}$ at time $\tau_{k}$. The equation of the characteristic line (assuming $q_{i, j}^{k}$ is constant across $\tau \in\left(\tau_{k-1}, \tau_{k}\right)$ ) that satisfies this condition may be expressed as,

$$
\mathscr{Q}_{i, j}(\tau)=Q_{j}+q_{i, j}^{k}\left(\tau-\tau_{k}\right)
$$

enabling us to discretise the derivative on the left-hand-side to read

$$
\frac{D V}{D \tau}=\frac{V_{i, j}^{k}-V\left(S_{i}, 2_{i, j}^{k-1}, \tau_{k-1}\right)}{\Delta \tau} .
$$

Crucially, if the point $\left(S_{i}, \mathscr{Q}_{i, j}^{k-1}, \tau_{k-1}\right)$ does not coincide with a node, a Lagrange cubic interpolation scheme is used to find the value $V\left(S_{i}, \mathscr{Q}_{i, j}^{k-1}, \tau_{k-1}\right)$. We can then rearrange the equation to all terms involving $q$ onto the right hand side along with the known values ( $V^{k-1}$ terms), so that the optimisation of $q$ may be performed before solving for $V^{k}$. We carry out the optimisation using a discretised linear search.

If we use $n$ nodes in $S$ and $m$ nodes in $Q$, then the result is a set of $(n \times m)$ linear equations for the $n \times m$ unknowns in $V$, and $n \times m$ objective functions that must maximised subject to admissible values of $q$. If we let $\tilde{q}$ to denote the optimal choice of $q$, then the full numerical scheme may be written as

$$
\begin{aligned}
\max _{q} f(q) & =V\left(S_{i}, \mathscr{Q}_{i, j}^{k-1}, \tau_{k-1}\right)+\Delta \tau\left(q S_{i}-\epsilon(q)\right), \\
V_{i, j}^{k}-\frac{\Delta \tau}{2} \mathscr{L}\left\{V_{i, j}^{k}\right\} & =\frac{\Delta \tau}{2} \mathscr{L}\left\{V_{i, j}^{k-1}\right\}+f(\tilde{q}),
\end{aligned}
$$


where $q \in[q, \bar{q}]$ and $\epsilon$ is a function of $q$. For a given timestep $\tau_{k}$ and $Q_{j}$, we must first find $\tilde{q}$ using (5.9) for all $S_{i}$, before we can solve the set of equations given by (5.10) for $V^{k}$ across all $i$ 's with a direct solver. The process can then be repeated at each timestep for each value of $Q_{j}$. Typically, the second order scheme will be accurate to around $0.01 \%$ across the $Q$ and $S$ space for a typical value of $\tau$ within in a time of order 10 seconds using an Intel Pentium 3.20 GHz CPU.

It is relatively straight-forward to include irreversible investment decisions into this numerical scheme. As discussed in Section 4.3, it involves comparing the valuation of different operational states (e.g. operational or abandoned) at each point $(i, j, k)$ within the solution domain, minus the investment cost to switch state. Where these two values smoothly meet determines the optimal boundary of transition. This concept has been widely used before within Real Options (e.g. Dixit \& Pindyck 1994), and we refer readers interested in the numerics of such switching decisions to Evatt et al. (2011) and Wilmott et al. (1995).

\subsection{Optimal valuation results}

We now compare our numerically determined optimal valuations and extraction regimes for three different forms of $\epsilon$, reflecting the fact that the cost form is imposed upon the system by the technology used by the resource owners. For comparison, we choose the same basic cost forms as before, i.e. (4.5), with $n=1,2,3$. In all the following experiments, we use the same parameter values as given in (4.17), but we must now include a time limit, which we choose to be $T=20 \mathrm{yr}$; one could interpret this to equivalently mean that the initial reserve size is $Q_{0}=2 \times 10^{7} \mathrm{~b}$. We have deliberately chosen this time and size limit, as it is also the time and size scales implied by (3.2) and (3.3) respectively, and thus we expect our analytical and numerical results to converge in this region of the time duration and resource size.

Figure 7 shows how the initial valuation of a resource subject to three cost functions: (4.5) with $n=3$ (dotted line), $n=2$ (dashed line) and $n=1$ (continuous line). As can be seen, these numerically derived valuations are all within $1 \%$ to the valuations calculated for a 'large enough' resource, shown in Figure 2. This is consistent with our expectations for a 'large enough' resource. However, it is important to see how the valuation converges to that for an infinite resource, as one varies the initial size of the resource.

To show the convergence of a finite resource valuation to an 'infinite' one, Figure 8 shows the valuation for different initial resource sizes (each assuming infinite contract duration $T$ ), all scaled to the fully perpetual solution (infinite contract duration and resource size). Five sets of results are shown, where the price is $S=\$ 120 \mathrm{~b}^{-1}$ and $\sigma=0.2 \mathrm{yr}^{-\frac{1}{2}}$. From lowest result upwards, $\delta=25 \%, 20 \%, 15 \%, 10 \%, 5 \% \mathrm{yr}^{-1}$. As such, we are able to observe how our 'large enough' size scale (3.3) works as we increase the convenience yield ${ }^{4}$. As the results clearly show, the location of the insensitivity to $Q$ increases near-linearly with $\delta$, confirming that that our choice of 'large enough' resource works well. Yet arguably the most important observation from this figure is that the valuation rapidly diminishes

4 We are interested in the sensitivity of our scaling for prices above break-even, as this is the price range in which companies shall commence extraction. 


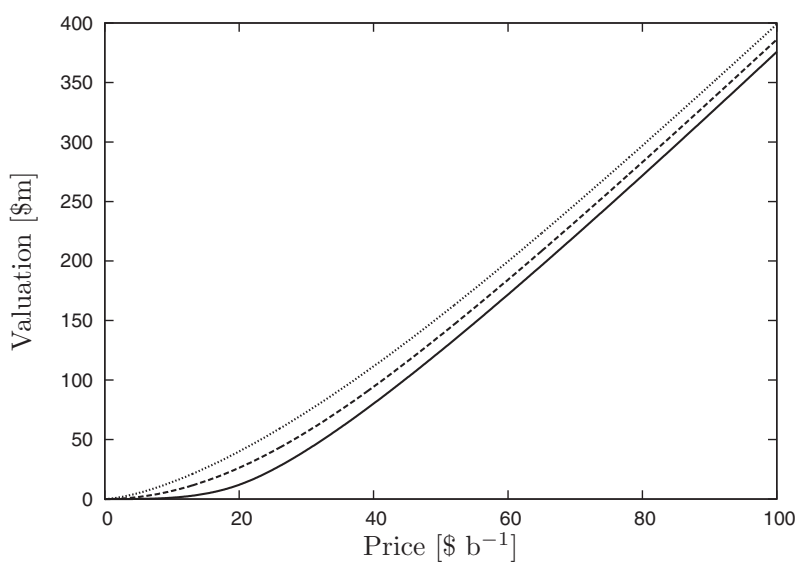

FIGURE 7. The optimal valuation of an extraction project whose cost function is a prescribed function of the extraction rate: $n=3$ (dotted line), $n=2$ (dashed line) and $n=1$ (continuous line). Solved using the numerical scheme of (5.9) and (5.10), with parameters given by (4.17).

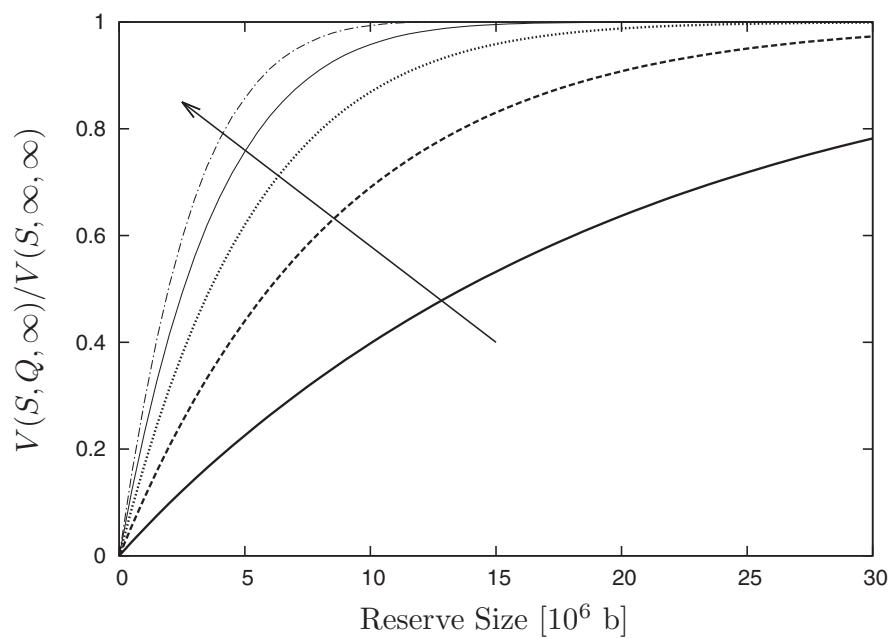

FIGURE 8. The valuation of an oil reserve with varying finite resource relative to the infinite resource case, with a quadratic cost function. The initial value of oil is $S=\$ 120 \mathrm{~b}^{-1}$ and $\sigma=0.2 \mathrm{yr}^{-\frac{1}{2}}$, and each line is for a different $\delta=5 \%, 10 \%, 15 \%, 20 \%$ and $25 \%$. The arrow indicates increasing $\delta$ and the contract length is perpetual.

for smaller resource sizes. This highlights the potential negative consequences of solving a resource valuation problem via an incorrect appropriate solution methodology; if the resource is smaller than the 'large enough' size scale, one must resort to numerical techniques in order to preserve any semblance of accuracy.

After examining our numerical valuations, we examine the associated optimal control strategies. The initial (time zero) optimal extraction rate regimes under the three values of $n$ are shown in Figure 9. This reaffirms that our analysis behind equation (4.4) is correct; 


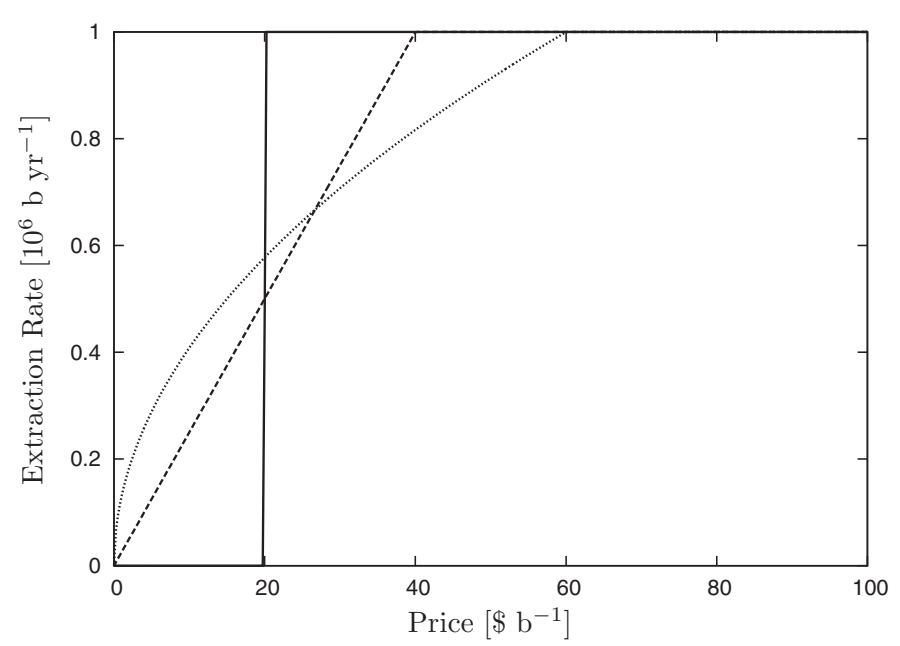

FIGURE 9. The initial optimal extraction rate of an oil reserve whose cost function is a prescribed function of the extraction rate; cubic (dotted line), quadratic (dashed line) and linear (continuous line). Solved using the numerical scheme of (5.9) and (5.10), with parameters given by (4.17).

the linear cost regime gives a jump-discontinuous optimal policy, the quadratic regime gives a near-linear policy, and the cubic cost regime gives a near-square-root policy. Again, these numerical results are consistent with the optimal policy for a 'large enough' resource, as shown in Figure 1. For these particular (taxless) parameter values, once the underlying price has reached $\$ 60 \mathrm{~b}^{-1}$, all extraction regimes are using the operator's maximum rate of output. The discontinuous extraction regime switches on at $20 \$ \mathrm{~b}^{-1}$, which is the lowest price at which the maximum extraction rate can have positive cash flows under a linear cost function. The fact that all optimal extraction regimes are extracting at $\bar{q}$ for all non-small values of $S$ explains why in Figure 7 the largest differences between the optimal valuation and the valuation at a constant extraction rate are at small values of $S$. The question then arises, how do such numerical control strategies vary in time?

By focusing upon the optimal quadratic control strategy, we examine how it converges to the control strategy in Figure 9 for different remaining levels of resource. Figure 10 shows the optimal control strategy for $n=2$, where the percentage of the remaining resource is, from top to bottom: $100 \%, 50 \%, 25 \%, 10 \%, 5 \%, 2.5 \%, 1.125 \%$ of the initial resource level, $Q_{0}$. It is clear that most variation in optimal strategy occurs during the latter stages of extraction, where significant deflection from the initial regime only occurs for (roughly) the remaining $25 \%$ of resource. Such a response in strategy is what one would expect, as this is consistent with the observation of the valuation rapidly converging to a constant value for large enough resources; the control strategy behaves likewise.

Figure 11 compares two numerical valuations, where the operation is subject to two forms of cost function: variable quadratic running costs of the form (4.5), and fixed running costs with $\epsilon_{r}=\$ 10^{7} \mathrm{yr}^{-1}$. However, in the upper valuation (continuous line), the operation can be abandoned altogether for an irreversible investment of $\$ 10^{7}$; the lower line (dotted) is the valuation where the operation can never abandon. In contrast to the 


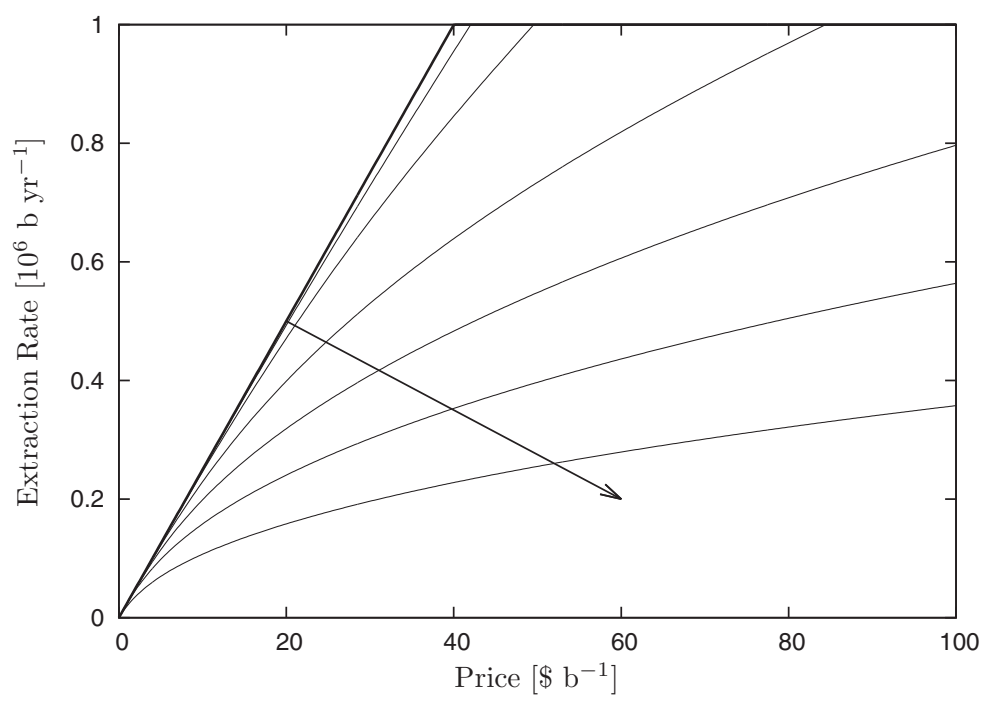

FIGURE 10. The numerically derived optimal extraction rate of an oil reserve versus price. The lines represent different remaining resource levels, and the solutions are made with no contract termination date (the perpetual case); in the direction of the arrow, the percentage of the remaining resource is $100 \%, 50 \%, 25 \%, 10 \%, 5 \%, 2.5 \%, 1.125 \%$ of the initial resource level, $Q_{0}$.

analytic abandonment solution shown in Figure 6, these valuations are calculated with an upper bound to the extraction rate. As can be seen, holding the option to abandon clearly improves the valuation of the operation, as this form of operation does not carry the likelihood of maintaining such significantly negative running costs as the no-abandonment case does. Indeed, this highlights the significant improvement in valuation which can be expected by combining and optimising irreversible and costless decisions; such a marrying of control strategies is both more representative of industrial practice and has been a long called-for area of investigation within Real Options, e.g. Brennan \& Schwartz (1985); Cherian et al. (2000); Kamrad \& Earnst (2001); Slade (2001).

Our final experiment compares two numerical valuations. The first valuation is made using the fully optimised numerically derived control strategy, whereas the second numerical valuation is made using the approximate optimal control strategy of a large enough resource (4.3), but maintaining its fixed structure over the entire finite lifetime of the mine. Using the quadratic extraction cost function, Figure 12 shows the percentage difference between the approximated and the exactly optimal valuations, for differing initial prices of the underlying. The results show a very small size of the error, which is a combination of three factors. Firstly, the variation in $V$ with respect to $Q$ in the exact valuation is small away from the final stages of extraction, making our control approximation, equation (4.3) reasonably accurate; secondly, the discounting of an already small difference in value from the final stages of extraction to the beginning of extraction reduces its effect on the valuation at the beginning of extraction; and finally, the fact that the optimal regime extracts at $\bar{q}$ for most values of $S$ means that differences between the accurate and the approximated optimal valuations can arise only over a small region of $S$. This high degree of accuracy may be of interest to practitioners who are hesitant to employ finite difference 


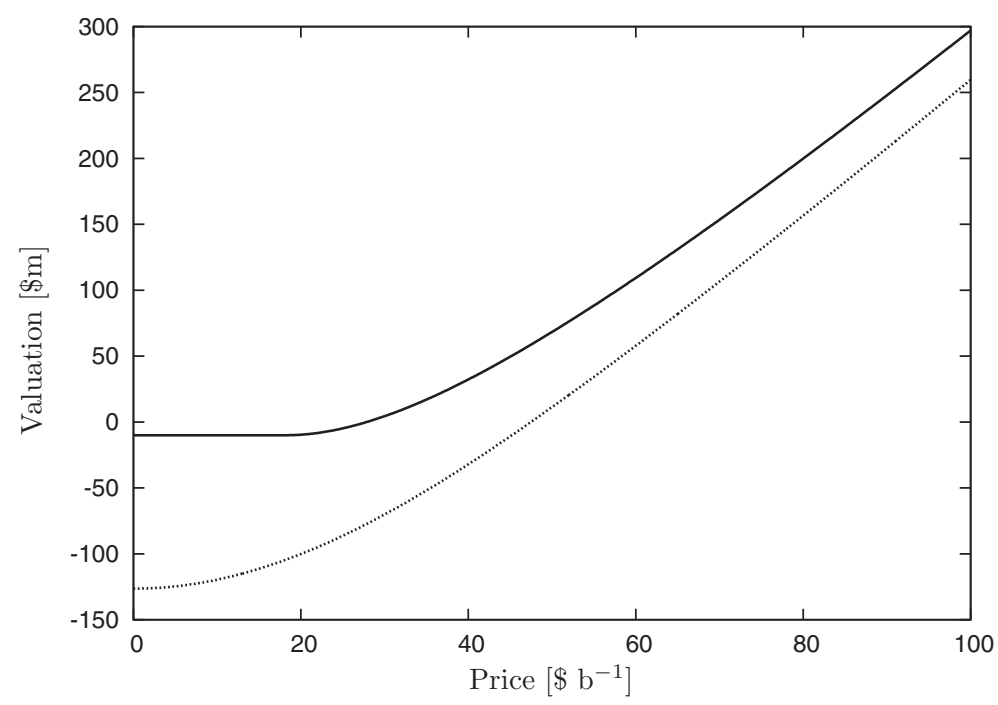

FigURE 11. Valuations for an extraction operation with both controllable (quadratic) costs and fixed running costs. The dotted line never has the option to abandon, whereas the continuous line may abandon the project when the price has reached a certain (optimal) level.

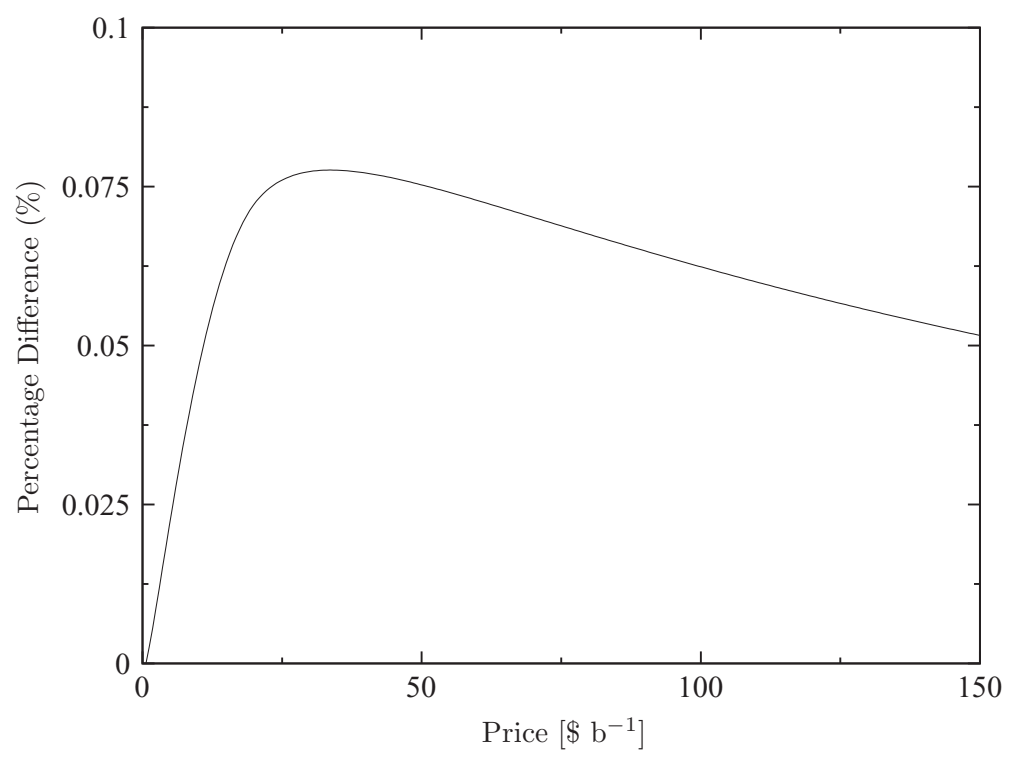

FiguRE 12. The percentage difference between the optimal extraction regime and approximate optimal extraction regime, for an oil reserve with quadratic cost function.

schemes, yet are able to use Monte Carlo methods. This is because the approximated control strategies of (4.3) can easily be placed within a Monte-Carlo scheme and thus provide a reasonable result for less computational effort. 


\section{Potential extensions}

Whilst we have considered a single uncertainty within a nonrenewable resource context, we now discuss how further uncertainties can be incorporated within our model, and how the work can be translated to a renewable resource extraction project.

\subsection{Additional uncertainty}

There are clearly many other uncertainties beyond price that could be included within a resource valuation (Atkinson \& Isangulov, 2010). These include interest rate variations, foreign exchange risks, geological estimation (Dimitrakopoulos \& Sabour, 2007) and operating cost uncertainty (Dehghani \& Ataee-pour, 2012). Precisely how these additional uncertainties affect the valuation and optimal control regime will clearly be more complex to calculate and heavily depend upon the nature of the uncertainty. When it is permissible to utilize some of these additional uncertainties within a more general form of the HJB equation (i.e. those which follow a Stochastic process), it is easy for us to gain qualitative insight into the likely behaviour of these systems. There are two particular cases one must be aware of: those in which the uncertainty is independent of the control variable, and those in which it is not.

In the first instance, let us suppose the resource valuation is exposed to an additional uncertainty $I$ which is independent of the control $q$, for example a foreign exchange rate. If suitable (as it often is with the mentioned risks), we could model $I$ via a general Ito diffusion of the form,

$$
d I=\mu_{I}(I, t) d t+\sigma_{I}(I, t) d X,
$$

where $\mu_{I}$ is the drift of the process and $\sigma_{I}$ is its volatility. With this generic form of uncertainty, the HJB equation of (2.5) can be extended (see Øksendal, 2003) to incorporate the process (6.1), to produce

$$
\frac{\partial V}{\partial t}+\mathscr{L}\{V\}+\mu_{I} \frac{\partial V}{\partial I}+\frac{1}{2} \sigma_{I}^{2} \frac{\partial^{2} V}{\partial I^{2}}+\rho \sigma_{I} \sigma \frac{\partial^{2} V}{\partial I \partial S}+\sup _{q}\left(-q \frac{\partial V}{\partial Q}+S q-\epsilon\right)=0
$$

where $\rho$ is the correlation between the price and $I$. Whilst the additional uncertainty has introduced further derivatives in $I$, these do not appear within the supremum term (because $\mu_{I}$ and $\sigma_{I}$ are not functions of $q$ ). Thus one may continue to use the approximate optimal control strategy of (4.3), where appropriate. As such, one can see that the form of the optimal control strategy (particularly for 'large enough' resources) may not be much affected by uncertainties independent of the control itself.

In the second instance, we consider a case when the parameters of some uncertainty $D$, are dependent upon the control $q$. Examples are variable extraction costs or volatile harvest rates. By writing this control-dependent uncertainty as

$$
d D_{t}=\mu_{D}\left(D_{t}, t, q\right) d t+\sigma_{D}\left(D_{t}, t, q\right) d X
$$


where $\mu_{D}$ is the drift and $\sigma_{D}$ is the volatility, our HJB equation now becomes

$$
\frac{\partial V}{\partial t}+\mathscr{L}\{V\}+\sup _{q}\left(\mu_{D} \frac{\partial V}{\partial D}+\frac{1}{2} \sigma_{D}^{2} \frac{\partial^{2} V}{\partial D^{2}}-q \frac{\partial V}{\partial Q}+S q-\epsilon\right)=0,
$$

where the new derivatives are contained within the supremum term (because the parameter is depend upon $q$ ). In general, this implies that finding an approximate optimal extraction regime is significantly more complex than before, even if the resource is 'large enough'. As such, one would have to revert to numerical techniques; one can use a variation on the scheme presented within Section 5, but the added dimension of $D$ means that in this case policy iteration would need to be carried out. However, there is one notable exception. This is when $D$ represents extraction cost uncertainty, and the parameters $\epsilon, \mu_{D}$ and $\sigma_{D}^{2}$ are all linear in $q$. In this particular instance, by differentiating (6.4) with respect to $q$ (as in Section 3), we see that the supremum term is independent of $q$ and thus the supremum is only found at the upper or lower bound of $q$. This in turn implies that a 'bang-bang' solution is optimal (although precisely where the control changes size still would require numerical calculation).

\subsection{Renewable resource control}

Let us now suppose that there is a rate of growth in the resource, $q_{i n}$, such as the growth of trees in timber forests (Morck et al., 1989; Provencher, 1995), rainfall flowing into a water reservoir (Roseta-Palma \& Xepapadeas, 2004) or a natural reproductive rate in fish stocks (Singh et al., 2006). In this instance, we must now extend the definition of the size of resource change from (2.2) to

$$
d Q_{t}=\left(q_{\text {in }}-q\right) d t+\sigma_{\text {in }} Q_{t} d W
$$

where we have allowed for the case that the growth rate is stochastic (similarly to Nøstbakken, 2006), and thus $\sigma_{\text {in }}$ represents the percentage volatility in the growth rate. Once again, we may incorporate this equation into the HJB equation to derive,

$$
\frac{\partial V}{\partial t}+q_{i n} \frac{\partial V}{\partial Q}+\mathscr{L}\{V\}+\frac{1}{2} \sigma_{i n}{ }^{2} Q^{2} \frac{\partial^{2} V}{\partial Q^{2}}+\sup _{q}\left(-q \frac{\partial V}{\partial Q}+S q-\epsilon\right)=0 .
$$

It is clear that the terms within the supremum are not altered from our original analysis, and thus one might again derive suitable approximations to the optimal extraction rate. However, a subtle distinction is introduced into what constitutes a 'large enough' level of resource. This is because the derivative in $Q$ is now multiplied by $\left(q_{\text {in }}-q\right)$, and thus it is possible for this term to be positive or negative. In either case, if this term is small, our definition of a 'large enough' resource (3.3) can become very small, because $\hat{q}$ in equation (3.1) would be representing the (averaged) difference between the growth rate and extraction rate.

To derive a numerical solution to this renewable situation (when one is retaining all of the derivatives) care must to impose the correct boundary conditions at the correct location. This is because with growth (or refilling), the characteristics can propagate in both a positive and negative direction, depending upon the sign of $q_{\text {in }}-q$. In the region 
for which $q_{i n}-q<0$, we would impose (as before) a boundary condition on $Q=0$. But, in the region of $q_{i n}-q>0$, we would need to prescribe a suitable problem-specific condition for when $Q$ became large. Further discussion of the direction of characteristics, in relation to gas storage, can be found in Chen \& Forsyth (2007).

\section{Conclusions}

This paper has examined the optimisation of extraction rates from a (representative) nonrenewable resource, where extraction rate changes are of a costless nature. This work will have main industrial application within the oil, gas and mining sectors (Schulze, 1974), where a significant level of research has already been considered in regards to irreversible investment decisions; costless rate-change decisions appear to have received much less detailed attention from within the related literature. Indeed, the importance (and complexity) of joining these two forms of decisions has been noted before, e.g. Brennan \& Schwartz (1985); Cherian et al. (2000); Slade (2001). With this in mind, we have explicitly shown how these two forms of decision can be married together to improve the overall efficiency of an extraction operation. As such, our analytical and numerical techniques relating to costless decisions, should be able to be embedded within many existing Real Options models, where the inherent complexity would appear to have previously hindered their inclusion.

We have performed our analysis via a PDE approach under the assumption of price uncertainty. Use of non-dimensionalisation and scaling arguments has allowed us to derive the conditions for when the level of remaining resource is 'large enough', that the associated valuation may be considered a close approximation to an infinite size resource valuation. As a consequence this size scale can be used as a simple test from when one is able to confidently use analytic approximations to find the optimal control strategy and corresponding valuation. Using this size assumption, we have provided closed-form solutions for a variety of cost functions. To complete the analysis, and fill a significant gap in the literature, we have also presented a numerical algorithm which can solve for the optimal strategy and valuation when the level of remaining resource is smaller than the 'large enough' size criterion.

\section{Acknowledgements}

This work was partially supported by the Centre for Interdisciplinary Control and Dynamical Analysis (CICADA), which is funded by the Engineering and Physical Sciences Research Council (EPSRC), UK, and partially by the SPRing project, also funded by EPSRC. We are grateful to Gemcom Software International (now Geovia) for their guidance regarding modern extraction projects, and to two anonymous referees for their helpful and detailed comments.

\section{References}

Ahn, C., Choe, H. J. \& Lee, K. (2006) A long time asymptotic behaviour for an american put. Proc. Am. Math. Soc. 137(10), 3425-3436. 
Atrinson, C. \& Isangulov, R. (2010) A mathematical model of an oil and gas field development process. Euro. J. Appl. Math. 21(03), 205-227.

Benchekroun, H., Halsema, A. \& Withagen, C. (2009) On nonrenewable resource oligopolies: The asymmetric case. J. Econ. Dyn. Control 33(11), 1867-1879.

Brennan, M. J. (1958) The supply of storage. Am. Econ. Rev. 48(1), 50-72.

BRENNAN, M. J. \& SChWARTZ, E. (1985) Evaluating natural resource investments. J. Bus. 58(2), 135-157.

Chen, Z. \& Forsyth, P. (December 2007) A semi-lagrangian approach for natural gas storage valuation and optimal operation. SIAM J. Sci. Comput. 30, 339-368.

Cherian, J. A., Patel, J. \& Khripko, I. (2000) Optimal extraction of nonrenewable resources when costs cumulate. In: M. J. Brennan \& L. Trigeorgis (editors), Project flexibility, agency, and competition, Oxford University Press, New York, pp. 224-253.

Cortazar, G., Schwartz, E. S. \& Salinas, M. (1998) Evaluating environmental investments: A real options approach. Manage. Sci. 44(8), 1059-1070.

Dehghani, H. \& Ataee-Pour, M. (2012) Determination of the effect of operating cost uncertainty on mining project evaluation. Resour. Policy 37(1), 109-117.

Deloitte (2011) Tracking the trends 2011. The top 10 issues mining companies will face in the coming year. Company report. http://www.deloitte.com/view/en_CA/ca/industries/ energyandresources/mining/efaa39766789c210VgnVCM3000001c56f00aRCRD.htm

Dimitrakopoulos, R. G. \& Sabour, S. A. A. (2007) Evaluating mine plans under uncertainty: Can the real options make a difference? Resour. Policy 32(3), 116-125.

Dixit, A. \& PINDYcK, R. (1994) Investment Under Uncertainty. Princeton University Press, New Jersey.

Dominy, S. C., Noppe, M. A. \& Annels, A. E. (2002) Errors and uncertainty in mineral resource and ore reserve estimation: The importance of getting it right. Explor. Min. Geol. 11(1-4), 77-98.

Evatt, G. W., Johnson, P., Duck, P., Howell, S. \& Moriarty, J. (2011) The expected lifetime of an extraction project. Proc. R. Soc. A: Math., Phys. Eng. Sci. 467, 244-263.

Evatt, G. W., Soltan, M. O. \& Johnson, P. V. (2012) Mineral reserves under price uncertainty. Resour. Policy 37, 340-345.

Hanemann, W. (1989) Information and the concept of option value. J. Environ. Econ. Manage. 16(1), 23-37.

KAMraD, B. \& EARnst, R. (2001) An economic model for evaluating mining and manufacturing ventures with output yield uncertainty. Oper. Res. 49(5), 690-699.

McCarthy, J. \& Monkhouse, H. L. (2002) To open or not to open: Or what to do with a closed copper mine. J. Appl. Corp. Finance 15, 63-73.

Moel, A. \& Tufano, P. (2002) When are real options exercised? An empirical study of mine closings. Rev. Financ. Stud. 15(1), 35-64.

Morck, R., Schwartz, E. \& Stangeland, D. (1989) The valuation of forestry resources under stochastic prices and inventories. J. Financ. Quant. Anal. 24(04), 473-487.

NøStbakken, L. (2006) Regime switching in a fishery with stochastic stock and price. J. Environ. Econ. Manage. 51(2), 231-241.

Øкsendal, B. (2003) Stochastic Differential Equations: An Introduction with Applications. Universitext (1979). Springer, Berlin, Germany.

PindYCK, R. (1988) Irreversible investment, capacity choice, and the value of the firm. Am. Econ. Rev. 78(5), 969-985.

Provencher, B. (1995) Structural estimation of the stochastic dynamic decision problems of resource users: An application to the timber harvest decision. J. Environ. Econ. Manage. 29(3), 321-338.

Roseta-Palma, C. \& Xepapadeas, A. (2004) Robust control in water management. J. Risk Uncertain. 29, 21-34.

SARKAR, S. (2000) On the investmentuncertainty relationship in a real options model. J. Econ. Dyn. Control 24(2), 219-225.

Schulze, W. D. (1974) The optimal use of non-renewable resources: The theory of extraction. $J$. Environ. Econ. Manage. 1(1), 53-73. 
SCHWARTZ, E. (1997) The stochastic behavior of commodity prices: Implications for valuation and hedging. J. Finance 52(3), 923-973.

Singh, R., Weninger, Q. \& Doyle, M. (2006) Fisheries management with stock growth uncertainty and costly capital adjustment. J. Environ. Econ. Manage. 52(2), 582-599.

Slade, M. (2001) Valuing managerial flexibility: An application of real-option theory to mining investments. J. Environ. Econ. Manage. 41(2), 193-233.

Staniforth, A. \& Cote, J. (1991) Semi-lagrangian integration schemes for atmospheric modelsa review. Mon. Weather Rev. 119, 2206-2223.

Wilmott, P. (2009) Paul Wilmott on Quantitative Finance, 2nd ed., Wiley, New Jersey.

Wilmott, P., Howison, S. \& Derwynne, J. (1995) The Mathematics of Financial Derivatives: A Student Introduction. Cambridge University Press, Cambridge, UK. 